\title{
Macrodipoles Unusual electric properties of biological macromolecules
}

\author{
Dietmar Porschke \\ Max-Planck-Institut für biophysikalische Chemie, D-37077 Göttingen, Germany
}

Received 28 April 1997; accepted 29 April 1997

\begin{abstract}
The wide range of different effects induced by electric fields in biological macromolecules is clearly due to the unusual quality and quantity of their electric parameters. A general concept for a quantitative description of the polarizability of macromolecules remains to be established. In the case of DNA, experimental data indicate the existence of an effective polarization length $\mathrm{N}_{p}$; at chain lengths $\mathrm{N}<\mathrm{N}_{p}$ the polarizability increases with $\mathrm{N}^{2}$, whereas saturation is approached at $\mathrm{N} \geq \mathrm{N}_{p}$. The polarization length decreases with increasing ionic strength in close analogy to the Debye length, but is $\sim 10$ times larger than the Debye length. The dynamics of DNA polarization at high field strengths has been observed in the ns time range and is consistent with biased field induced ion dissociation. In the range of chain lengths from $\sim 400$ to $\sim 850$ base pairs DNA molecules exhibit permanent dipole moments, which are in a preferentially perpendicular direction to the end-to-end-vector, leading to a positive electric dichroism. These results are consistent with a "frozen" ensemble of bent DNA configurations and provide evidence for the existence of slow, non-elastic bending transitions. The electric parameters of proteins are usually dominated by a permanent anisotropy of the charge distribution, corresponding to permanent dipole moments of the order of several hundred Debye up to about 1500 Debye. Relatively small dipole moments of protein monomers add up to millions of Debye, when these proteins are in a vectorial organization in membrane patches, as found for bacteriorhodopsin and $\mathrm{Na}^{+} / \mathrm{K}^{+}$-ATPase. In these cases the dipole vector may support vectorial ion transport. It is remarkable that the dipole moments of proteins usually show a relatively small dependence on the salt concentration; a rational for these observations is provided by a dipole potential at the plane of shear for rotational diffusion, which is defined in close analogy to the $\zeta$-potential for translational diffusion. Symmetry breaking leading to huge electric dipole moments may be expected for mixed lipid vesicles: according to model calculations the phase separation of lipid components with and without net charges may lead to very high dipole moments; the expectation has been verified experimentally for vesicles containing DMPA and DMPC. The state of these systems should be extremely sensitive to electric fields. In summary, there is an unusual wide variation of electric parameters associated with biological macromolecules and with biomolecular assemblies, which is the basis for the complexity of different phenomena induced by electric fields in biological systems. (C) 1997 Published by Elsevier Science B.V.
\end{abstract}

Keywords: Polarizability; permanent dipole moment; proteins; nucleic acids; lipids 


\section{Introduction}

The effects of electric fields on biological macromolecules is of considerable interest for various reasons. First of all, processing of electrical signals in biological systems is based on the interactions of proteins or other macromolecular components with electric fields and on reactions induced by these fields. Thus, a qualitative and quantitative understanding of bioelectricity requires knowledge of the electric parameters of the molecules involved in these processes. Furthermore, electric fields are widely used for the analysis of biological macromolecules. In spite of the considerable practical importance, a quantitative description of the effects induced by electric fields on these macromolecules is usually not available. Finally, there is a serious uncertainty on the potential effects of low electric fields on biological systems. Although it is quite obvious that there is hardly any problem under usual environmental conditions, it is also known that some organisms show a remarkably high sensitivity towards electric fields [1]. This raises the question on the physical basis and the limits for such sensitivity [2].

The analysis of effects induced in macromolecules by electric fields has a long tradition. Part of the work reported in the present review is based on the developments by Manfred Eigen and his coworkers during the golden age of chemical relaxation in the $50^{\prime}$ and $60^{\prime}$ of this century [3-4]. The progress since then is based on several developments. First of all it has been possible to increase the sensitivity of the experimental techniques. A serious difficulty in experimental studies was the unequivocal separation of chemical and physical effects induced by electric fields (cf. [5]); it is quite obvious that these effects were not always separated successfully in early studies. Another important factor is the availability of macromolecules in a sufficient quality and quantity: for example, the possibility to prepare DNA restriction fragments, providing monodisperse polymer molecules of various, well defined length, has been very useful for a quantitative analysis of the electric polarizability of polyelectrolytes. Finally, the development of computers has provided tools for numerical analysis, which prove to be particularly useful for the analysis of the complex processes induced by electric fields in polymers. Because of the large numbers of charged residues in most biopolymers and because of the coupling of many different effects induced by electric fields, most of the field induced phenomena cannot be described in terms of simple analytical procedures and require numerical simulations.

The literature on the subject of the present review is extensive and, thus, the review cannot be complete. The main emphasis is on recent developments, which appeared to be of interest to the author.

\section{Theory}

Electric fields induce rotational motion of molecules with dipole moments, moving their dipole vector into the direction of the field vector. Most molecules of interest also show some optical anisotropy; under these conditions the field induced rotational motion may be detected conveniently by measurements of the optical anisotropy. The quantitative description of field induced rotation detected by the optical anisotropy requires knowledge on the hydrodynamic, electrostatic and optical parameters of the molecules under investigation. The following short sections on these subjects are very brief introductions, which should provide at least some theoretical background on the procedures used in the following sections.

\subsection{Hydrodynamics}

The shape of some macromolecules may be approximated by spheres or ellipsoids, but these simple models cannot be used in the majority of the cases. For objects with a complex shape the hydrodynamic parameters may be simulated by the following "bead model simulation" procedure [6]: 1.) the overall shape of the object is approximated by an assembly of spherical "beads"; 2.) the hydrodynamics of cach bead is described by Stokes law; 3.) the hydrodynamic coupling between each bead is described by a special coupling tensor. Obviously the description of the hydrodynamic coupling is the critical step. Appropriate coupling tensors have been developed [6]; tests have demonstrated that the results obtained by bead model simulations are quite reliable [7].

In the context of macrodipoles, bead model simulations are used, for example, to define the center of diffusion, which serves as the origin of the coordi- 
nate system for the appropriate calculation of dipole moments in the case of molecules with a net charge [8-9].

\subsection{Optical parameters}

The anisotropy of light absorption is described by extinction coefficient tensors. In the case of macromolecules there are often many different residues contributing to the absorbance. If the extinction coefficient tensors of the residues are known, the tensor of the complete molecule is calculated by tensorial addition: the components have to be represented in a reference coordinate system and then can be added together.

\subsection{Electric parameters}

As discussed in various sections of this review, permanent dipole moments $\mu_{p}$ may be calculated relatively easily according to

$$
\mu_{p}=\sum_{i} q_{i} r_{i}
$$

provided that the coordinates $r_{i}$ of the charges $q_{i}$ on the charged residues are known. If the crystal structure is known, the coordinates can be obtained from appropriate data banks, e.g. the Brookhaven Protein Data Bank. If the structure has not been determined yet, it is possible to construct models, using the methods of molecular modelling.

The calculation of polarizability tensors is far more difficult and continues to be a challenge even for polyelectrolytes of relatively simple shape (cf. section 3.3.).

\subsection{Calculation of transients}

If the hydrodynamic, optical and electric parameters are known, it is possible to calculate electrooptical transients. For example, dichroism decay curves, measured at zero field strength, may be calculated using the equations given by Wegener, Dowben and Koester [10]. Wegener [11] has also described the calculation of electrooptical risecurves in the Kerr limit of low electric fields; these risecurves involve 8 relaxation times in the general case.
In practice it is rarely possible to keep the electric field strength within the Kerr limit. For the case of arbitrary electric field strengths, transients may be calculated by Brownian dynamics simulations [12]. This approach proves to be very useful for the characterization of electrooptical risecurves.

\section{Polarizability of polyelectrolytes}

Most biopolymers have a high charge density and are surrounded in solution by an extensive ion atmosphere. When these polymers are exposed to external electric fields, the polymer molecules start to react, e.g. by alignment into the direction of the field vector, already at particularly low field strengths, indicating the existence of an unusually high electric polarizability. There is a large number of different biopolymers, which may be used for investigations of the unusual electric parameters. However, double helical DNA has been studied much more extensively than any other biopolymer for various reasons. One of the arguments for investigation of DNA is of course its biological importance. Several technical arguments for DNA are based on its molecular properties: the double helix is optimal for investigations a) because of the well defined structure; b) because of the unusually high optical anisotropy resulting from stacking of the bases in an almost perpendicular direction with respect to the long axis; c) because of the availability of well defined fragments of homogeneous molecular structure over a broad range of chain lengths. As soon as experimental studies on DNA restriction fragments were available, most of the data accumulated in the early literature for various polydisperse macromolecules were not of much interest anymore. The data obtained for DNA restriction fragments are very useful for comparison with a wide variety of theoretical models, which have been developed over the years by many different groups (for a recent model with references to the literature cf. [13]).

\subsection{The polarizability of DNA, its dependence on the chain length and the ionic strength}

The field induced orientation of DNA can be recorded relatively easily by measurements of the 
dichroism (or of the birefringence). The magnitude of the dichroism measured at different electric field strengths may then be compared with orientation functions [14], which describe the degree of orientation by a Boltzmann function involving the energy of a given type of dipole in the electric field relative to the thermal energy. An analysis of the data obtained for DNA indicated that its electric parameters cannot be explained by a simple induced or permanent dipole function. The experimental data obtained for double helices at low field strengths are consistent with an induced dipole, whereas at high field strengths the data are consistent with a permanent dipole. Thus, it has been concluded that the polarizability of DNA saturates [15-19]. According to the experimental data, the electric field strength, where the polarizability approaches saturation, decreases with increasing chain length [16-17]. The saturation will not be discussed here; the following short discussion is restricted to the polarizabilities $\alpha$ measured at low field strengths.

The polarizabilities of DNA restriction fragments have been characterized by different groups. Unfortunately the conclusions are different with respect to the dependence on the chain length $\mathrm{N}$ : according to one of the reports [20] the polarizability increases with $\mathrm{N}^{3}$ at low $\mathrm{N}$-values, whereas two other groups [16-17] found an increase with $\mathrm{N}^{2}$. Contradictions of this type are clcarly not optimal for the field, because it remains unclear where the difference comes from.

It must be expected that the chain length dependence is affected by the length scale describing electrostatic interactions at the given solution conditions. Thus, the increase of the polarizability with the chain length, which is found at low $\mathrm{N}$-values, is expected to be reduced at higher chain lengths due to electrostatic shielding. Another effect contributing to the observed chain length dependence is the inherent flexibility of the chains. Data from the authors laboratory indicate that the increase of the polarizability $\alpha$ with the chain length $\mathrm{N}$ approaches saturation at high chain lengths. This dependence may be described by the following exponential function

$$
\alpha=\alpha_{\infty}\left(1-e^{-\left(N / N_{p}\right)^{2}}\right)
$$

where $\alpha_{\infty}$ represents the polarizability in the limit of infinitely high chain length $\mathrm{N}$ and $\mathrm{N}_{p}$ represents

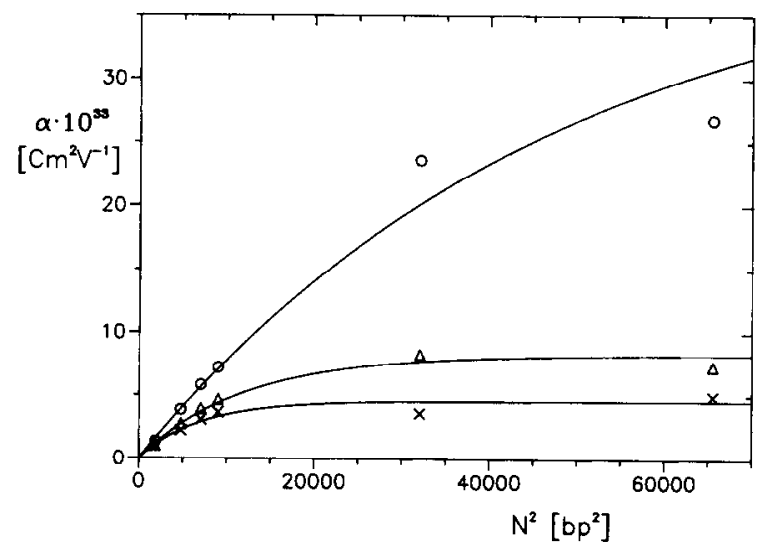

Fig. 1. Polarizability $\alpha$ of DNA restriction fragments as a function of the square of the chain length $\mathrm{N}^{2}$ at different ionic strengths: $2.44 \mathrm{mM}(\mathrm{o}), 7.44 \mathrm{mM}(\triangle)$ and $12.44 \mathrm{mM}(x)$. The continuous lines represent least squares fits according to equation (2); the resulting parameters are compiled in table 1 .

an effective polarization length. At low chain lengths the polarizability described by equation (2) increases with the square of the chain length according to

$$
\alpha=\alpha_{\infty}\left(N / N_{p}\right)^{2}
$$

According to least squares fits of experimental data to equation (2) the limit chain length $\mathrm{N}_{p}$ decreases strongly with increasing ionic strength of the solution (figure 1, table 1). It is remarkable that the magnitude of $\mathrm{N}_{p}$ is clearly larger than the Debye length $\mathrm{r}_{d}$. However, the decrease of $\mathrm{N}_{p}$ with increasing ionic strength is closely related to the corresponding ionic strength dependence of the Debye length. As shown in table 1, the ratio of the effective polarization length and the Debye length $r_{d}$ is virtually independent of the ionic strength and is about 11 . The limit slope $\alpha_{\infty} / \mathrm{N}_{p}^{2}$ observed in the range, where $\alpha$ is a quadratic function of $\mathrm{N}$, shows a relatively small decrease with increasing salt concentration (cf. table 1).

Stellwagen [16] and Elias \& Eden [20] presented their chain length dependence of the polarizability without a discussion of a limit chain length. This appears to be mainly due to the fact that these authors measured at a lower ionic strength, where the limit chain length is larger and an approach to saturation is not clearly indicated by the experimental data. The polarizabilities presented by Diekmann et al. [17] are in very satisfactory agreement with equation (2): 
Table 1

Comparison of the polarization length $\mathrm{N}_{p}$ obtained according to equation (2) with the Debye length $\mathrm{r}_{d}$ and the limit slope of the polarizability according to equation (2).

\begin{tabular}{cccccc}
\hline Ionic Strength & $\mathrm{r}_{d}$ & $\mathrm{~N}_{p}$ & $0.34 \times \mathrm{N}_{p}$ & $0.34 \times \mathrm{N}_{p} / \mathrm{r}_{d}$ & $\alpha_{\infty} /\left(\mathrm{N}_{p}\right)^{2}$ \\
\hline$[\mathrm{mM}]$ & {$[\mathrm{nm}]$} & {$[\mathrm{bp}]$} & {$[\mathrm{nm}]$} & - & $\mathrm{Cm}^{2} \mathrm{~V}^{-1} \mathrm{bp}^{-2}$ \\
\hline 2.44 & 6.17 & 219 & 74.3 & 12.0 & $8.6310^{-4}$ \\
7.44 & 3.53 & 110 & 37.2 & 10.5 & $6.9210^{-4}$ \\
12.4 & 2.73 & 84 & 28.6 & 10.5 & $6.5110^{-4}$ \\
\hline
\end{tabular}

the $\mathrm{N}_{p}$-value is virtually identical with that given for the lowest ionic strength in table 1 , whereas the limit slope is lower. Rau and Charney [21] discussed in a theoretical paper, that "the ends of the rod become increasingly isolated, electrostatically" with increasing chain length and concluded that the dependence of the polarizability on the chain length changes from a quadratic to a linear one at high chain lengths. However, these authors did not present experimental data.

\subsection{The dynamics of polarization}

Independent information about the polarization process may be obtained by an analysis of its dynamics. A first report on the measurement of a polarization time constant of DNA was based on the frequency dependence of the anisotropy of the conductivity $[22,23]$. According to these measurements the polarization time constant of DNA with $\sim 1400$ base pairs is $0.3 \mu \mathrm{s}$. The authors have described the frequency dependence of the orientation by a single relaxation time $\tau_{0}$. The authors claim that the sensitive dependence of all quantities, including $\tau_{0}$, on the cubic power of the length has been established. It is obvious that these early results had a strong impact on the field. However, an examination reveals clear problems, which appear to be mainly due to the fact that in those early days the physical properties of DNA were not known in sufficient detail. First of all, the cubic power dependence has been suggested by a simple model, but has not been checked experimentally. The description of the frequency dependence of the orientation by a single relaxation process is oversimplified; it has been clearly demonstrated by different groups that there is more than a single relaxation process for double helices with $\sim 1400$ base pairs (for measurements in the frequency domain cf.
$[24,25])$. The chain length of the DNA was far beyond the persistence length; it is known that in this range there are special contributions to field induced orientation (e.g. section 4.). Finally, technical problems in the early measurements are indicated by a clear dependence of the relaxation time of "disorientation" on the DNA concentration. In summary, the early investigation was a useful stimulus; however, part of the results, including the polarization time constant, require reinvestigation.

More than 20 years later the dynamics of DNA polarization has been analyzed again by measurements of dichroism risecurves at a very high time resolution [26]. An unequivocal assignment required use of DNA restriction fragments; the chain lengths were in a range below 100 base pairs, where the helices behave like rigid rods and their rotational diffusion can be described by single time constants. The quantitative analysis of dichroism risecurves measured for DNA fragments with 76 and 95 base pairs, based on a deconvolution procedure, revealed a small but clearly reproducible delay of the dichroism rise. This type of delay must be expected, because straight DNA double helices are without dipole before application of a field pulse. The dipole is created upon field application. The quantitative analysis of the dichroism risecurves showed that these risecurves are convolution products of the polarization process and the rotational diffusion process. The polarization time constants $\tau_{p}$ are in the range of $\sim 10 \mathrm{~ns}$. Measurements at different ion concentrations revealed that $1 / \tau_{p}$ is a linear function of the salt concentration, as may be expected for simple binding of cations to the negatively charged DNA. The rate constant of cation binding, which is derived from this dependence, is $\mathrm{k}^{+} \sim 10^{10} \mathrm{M}^{-1} \mathrm{~s}^{-1}$; this value indicates a diffusion controlled process of cation binding to the DNA, which is consistent with 
expectation and suggests that both data and interpretation are reasonable. The rate constants of cation dissociation $\mathrm{k}^{-}$have also been derived from the experimental dependence of $\tau_{p}$ on the salt concentration; the $\mathrm{k}^{-}$-values increase with increasing electric field strength, indicating field induced dissociation of cations from the DNA. Thus, according to the most simple interpretation of the experimental data, the dipole moment is generated by biased dissociation of cations from one of the ends of the double helix.

It should be noted that the polarization time constants obtained for the two fragments with 76 and 95 base pairs were found to be identical, within the limits of experimental accuracy. If the polarization time constant would increase with the cube of the chain length, as suggested by certain models, the time constants should differ by a factor of $\sim 2$. A difference of this magnitude should have been noticed and, thus, the available data provide evidence against a dependence on the cube of the chain length. However, it should be noted that there may be different regimes of polarization mechanisms (cf. [26]).

\subsection{Brownian dynamics simulations}

A theoretical analysis of the polarization of polyelectrolytes is very difficult, because many different effects have to be considered and it is virtually impossible to include all these effects in an analytical treatment. Under these conditions Brownian dynamics simulations appear to be a useful approach to the problem $[27,28]$. Although it is not possible to describe the details of such simulations in the present context, it should be useful to discuss at least some of the results.

The model is a simple rodlike polymer within a box surrounded by counterions and eventually by additional ions [28]. The electrostatic interactions between the particles in the box are calculated under conditions equivalent to those in aqueous solution. Interactions beyond the box are evaluated by Ewald summation assuming periodic boundary conditions. Application of electric field pulses to this system leads to the generation of a dipole moment parallel to the field vector with time constants close to 10 ns. These simulated time constants are in satisfactory agreement with the experimental polarization time constant obtained from dichroism risecurves (cf. previous section).

The Brownian dynamics simulations demonstrate, for example, that there is field induced dissociation of ions from the polyelectrolyte. The simulations also show that the dipole moments saturate at high field strengths. There are many more effects that can be demonstrated by these simulations; some of these can hardly be verified by experimental studies. For example, experimental data provide only the overall dipole moment corresponding to the difference between that induced parallel and perpendicular to the long axis of DNA. In the Brownian dynamics model it is no problem at all to simulate both components separately. According to these simulations the perpendicular component is surprisingly close to the parallel one, at least for helix models up to 30 base pairs length. The simulated data indicate that the parallel component increases more than linearly with the chain length, whereas the increase of the perpendicular component is linear with the chain length.

A major problem in the assignment of polarizabilities is the effect of hydrodynamic interactions (cf. [29]). Recent simulations [30] demonstrate that the influence of hydrodynamic interactions is rather small for polymers with counterions in the absence of byions. It has been shown that addition of byions leads to a considerable increase of parallel dipole components due to hydrodynamic interactions. Thus, the Brownian dynamics approach can be used to characterize individual contributions to the polarizability of polyelectrolytes.

\section{A special type of 'permanent' dipole in DNA double helices}

A large number of different laboratories have studied the electric dichroism of DNA. The general conclusion from all these investigations was that the electric dichroism of DNA double helices is negative. Occasionally there were some reports on unusual transients for DNA samples showing a relaxation component with a positive amplitude; usually these observations were attributed to special conditions and have not been analyzed in detail. A positive stationary value of the electric dichroism of DNA has not been reported before 1994 .

During a control experiment in the authors labo- 
ratory it was found by accident that the stationary dichroism of a given DNA restriction fragment is positive under special conditions [31]. The conditions of the experiments were unusual in the sense that the electric field strength applied to the solution was much lower than used previously. The detection of optical signals under these conditions required an instrument with a particularly high signal to noise ratio. Extensive measurements confirmed the existence of a regime, where the electric dichroism of double helical DNA is positive; it could be demonstrated that this effect is not just an artifact and that it is not caused by aggregation or by an unusual conformation of the DNA.

The positive electric dichroism was detected at low electric field strengths $E \leq 1 \mathrm{kV} / \mathrm{cm}$ in a range of chain lengths between approximately 400 and 850 base pairs (figure 2); the buffers used for these measurements contained monovalent ions at ionic strengths between 1 and $11 \mathrm{mM}$. In all cases the positive dichroism turned to the standard negative one, when the electric field strength was increased to values above $\sim 1 \mathrm{kV} / \mathrm{cm}$ (figure 2). Apparently the electric field strengths used in previous investigations reported in the literature was $>1 \mathrm{kV} / \mathrm{cm}$.

The electrooptical analysis at low field strengths [31] revealed another unusual feature: the positive electric dichroism always appeared together with transients indicating permanent dipole moments. When the electric field strength $\mathrm{E}$ was increased, the transients typical of permanent dipoles were converted into transients known for induced dipoles (figure 2); this conversion was observed in the same range of E-values, where the stationary dichroism is converted from positive to negative values. Because all these effects are observed at very low electric field strengths, it is clear that the permanent dipole moment must be rather large. An approximate value for the dipole moment has been evaluated by an analysis of the relaxation amplitudes obtained from dichroism risecurves. The amplitudes corresponding to the positive dichroism have been measured at different E-values and have been subjected to least squares fitting according to the orientation functions for permanent and induced dipoles. Fitting was successful using the permanent dipole function with a value for the permanent dipole of $\sim 70000$ Debye.

The observation of a positive dichroism and a large

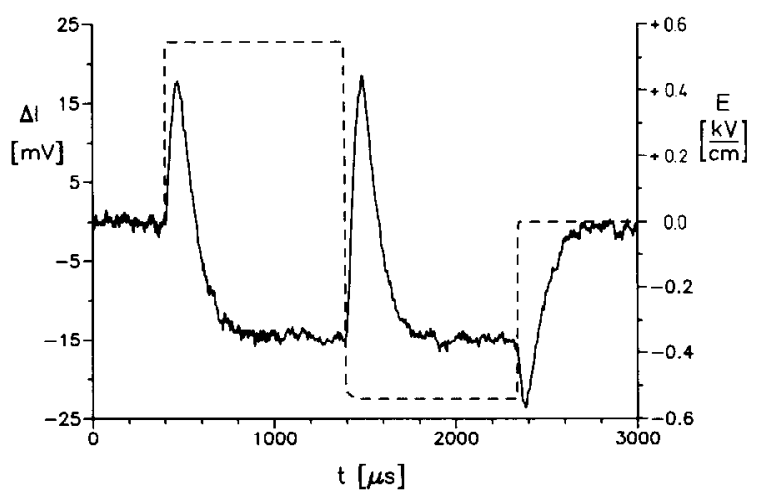

(a)

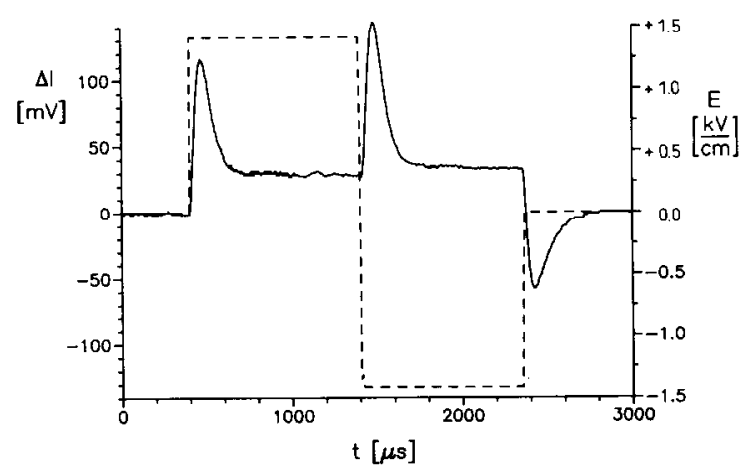

(b)

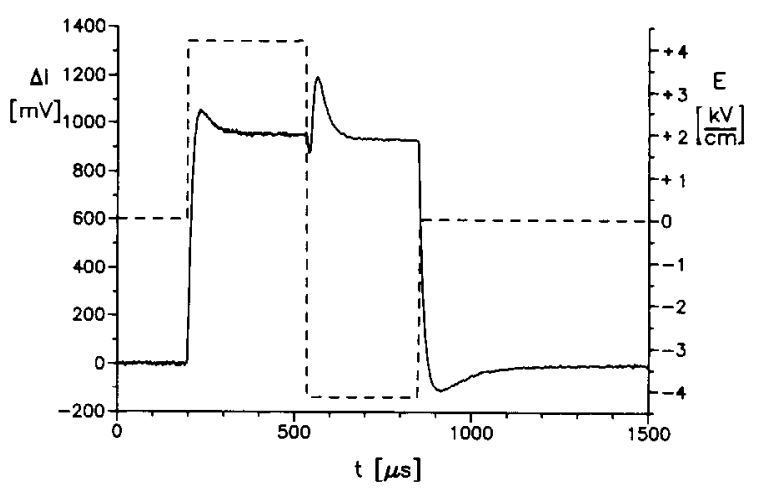

(c)

Fig. 2. Change of the light intensity $\Delta I$ transmitted through a solution of a 587 bp fragment as a function of time $t$ during exposure to electric field pulses (dashed lines) of 3 different electric field strengths $\mathrm{E}$. The light is polarized parallel to the electric field vector ( $1 \mathrm{mM} \mathrm{NaCl}, 1 \mathrm{mM} \mathrm{Na-cacodylate} \mathrm{pH} 7.0$, $0.2 \mathrm{mM}$ EDTA at $2^{\circ} \mathrm{C}$; DNA helix concentration $0.121 \mu \mathrm{M}$; from [31]). 

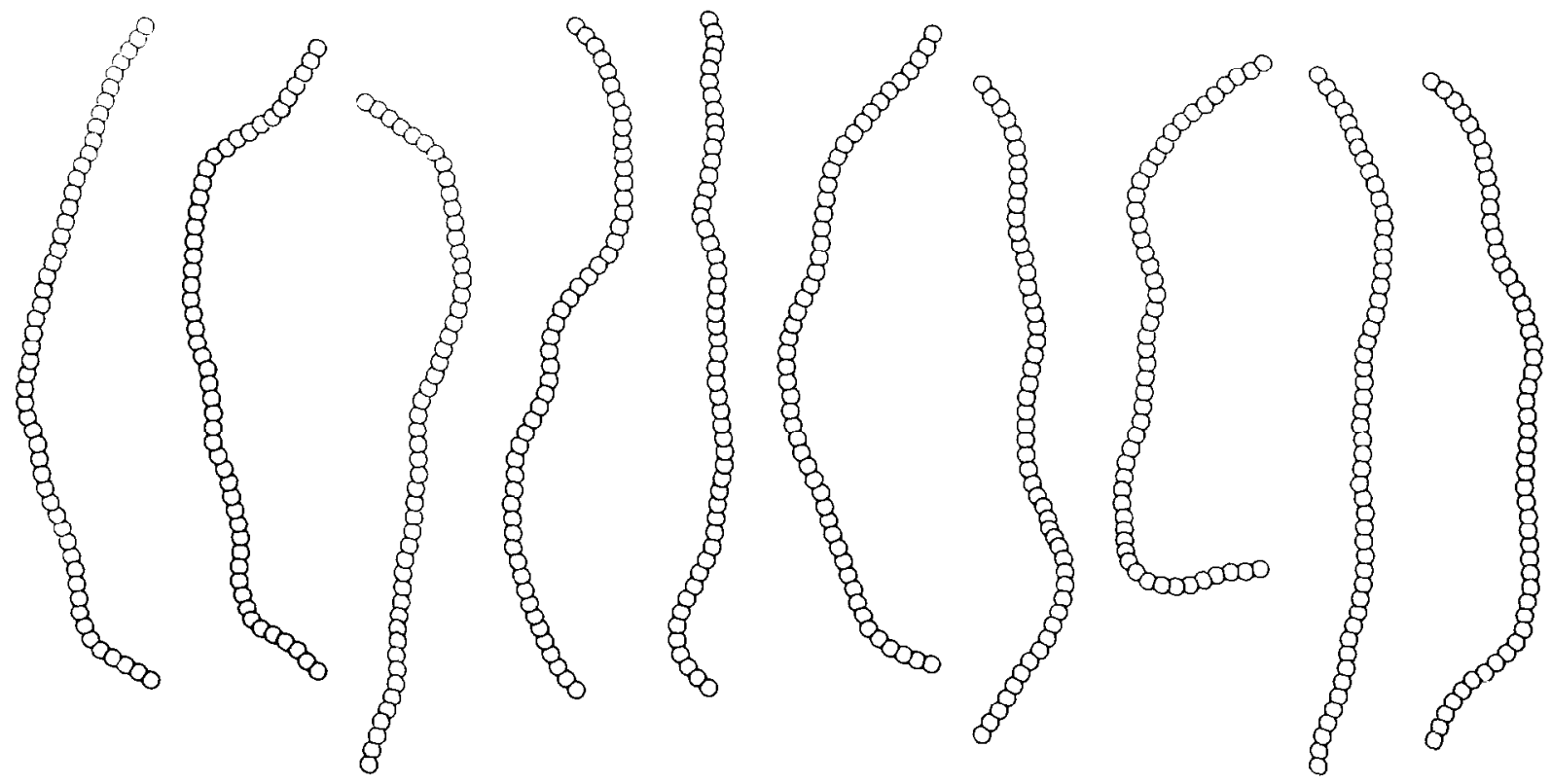

Fig. 3. Configurations generated according to the wormlike coil model for a contour length corresponding to the persistence length (from [31]).

permanent dipole for DNA under conditions, where this DNA is expected to be in its standard double helical form, raises serious questions. It cannot be excluded that the DNA is transformed to an unusual structure, which has not been characterized until now. However, there is an explanation of the unusual observations, which is based on the standard structure and known parameters of this structure. First we have to consider the fact that the positive dichroism has been observed in a range of chain lengths, where the inherent flexibility of the double helix leads to clear deviations from the straight form. Inspection of a set of configurations generated by a Monte Carlo procedure (figure 3 ) shows that virtually all of the configurations are not symmetric. For highly charged molecules like DNA, loss of symmetry implies the existence of an electric moment.

The standard definition of dipole moments according to physics has been given for molecules without net charge. In the case of molecules like DNA with a high negative net charge, the standard definition cannot be used; however it is clear that in the presence of an external electric field molecules with a non-symmetric distribution of charges experience a torque. The appropriate extension of the concept of dipole moments to this special situation requires calculation of the electric moment to a special reference point: the center of diffusion. The coordinates of the center of diffusion within the coordinate system of a given molecular configuration may be obtained by bead model simulations (cf. 2.1). For most of the configurations shown in figure 3 the charge distribution with respect to the center of diffusion results in a dipole moment with a preferential direction which is perpendicular to the end to end vector. This direction of the dipole moment implies a positive value of the electric dichroism.

Obviously there is a broad distribution of configurations and thus also a broad distribution of values of the dichroism. One of these distributions for a given chain length and persistence length is illustrated by a population diagram in figure 4 . It is very obvious that the majority of the configurations is associated with a positive dichroism. The calculations leading to the results presented in figure 4 are based on the assumption that the lifetime of each of the configurations is sufficiently long compared to the time base of the experiment. Thus, the experimental data indicate the 


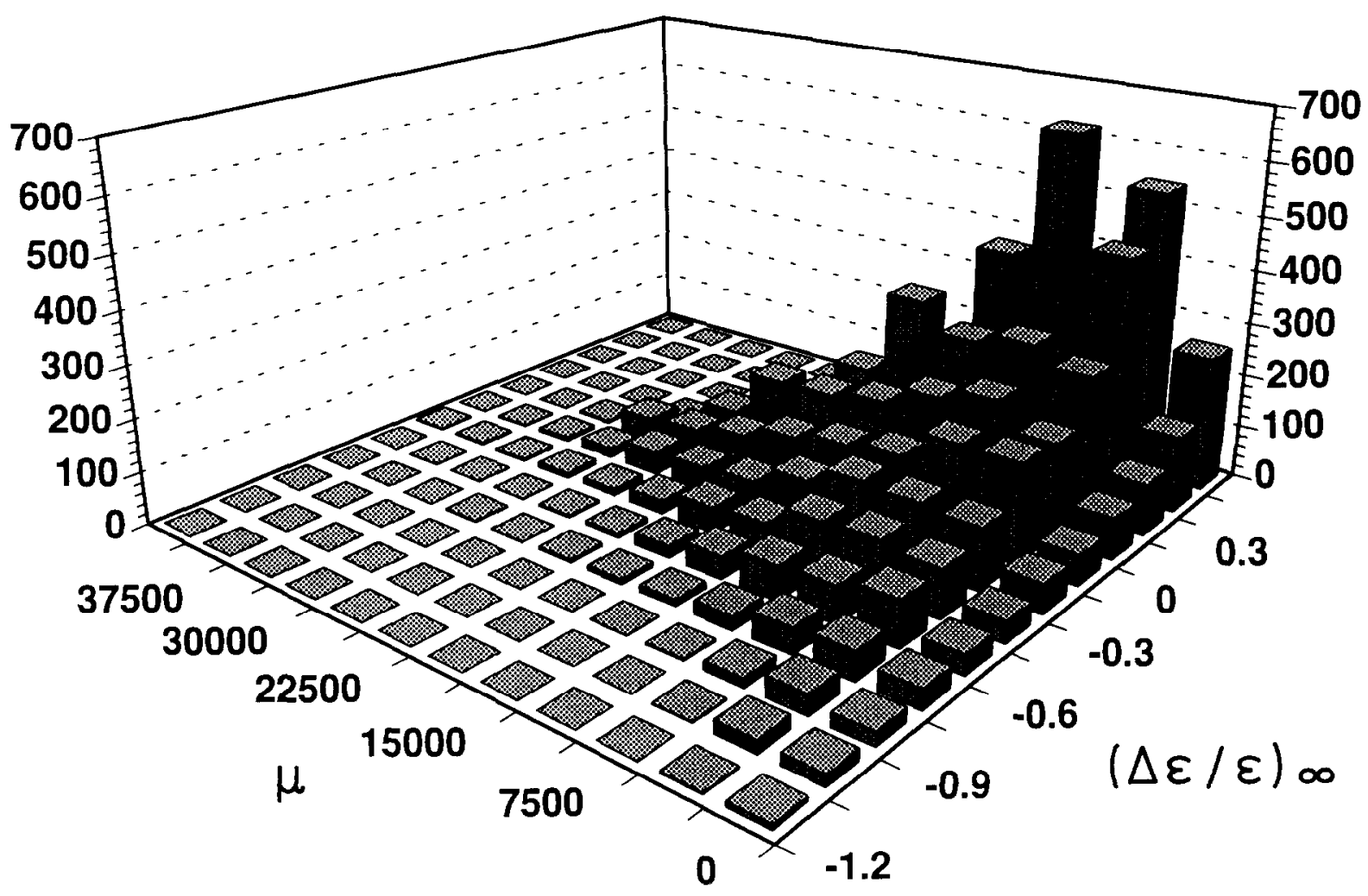

Fig. 4. Population diagram of bent DNA molecules generated by a Monte Carlo procedure: Number of different configurations found in different ranges of the dipole moment $\mu$ and of the limit electric dichroism $(\Delta \epsilon / \epsilon)_{\infty}$ from a simulation for a DNA double helix with 587 bp with a total number of 8000 random configurations; persistence length $4500 \AA$; the average limit dichroism is +0.0863 ; the stationary reduced dichroism (at low field strengths) is $2.43 \times 10^{-3}$; the average decay time constants are $\tau_{1}=6.3 \mu \mathrm{s}, \tau_{2}=72.2$ $\mu \mathrm{s}$; the maximal dipole moment in the ensemble is $43460 \mathrm{D}$ (from [31]).

existence of a "frozen" ensemble of configurations. In this context "frozen" refers to the time scale of the electrooptical experiments (cf. figure 2), which are conducted in the $\mu \mathrm{s}$ time range; thus, the configurations are converted into each other at a slower time scale.

Some further observations [31] add evidence for the interpretation of the data described above. The positive dichroism proved to be quite sensitive to photoreactions: a relatively small dose of UV-radiation is sufficient to convert the positive dichroism to a negative one. It is known that UV-irradiation of DNA leads to the formation of various products, which tend to interrupt base stacking, which is the main source of the mechanical rigidity of the double helix. Thus, the ensemble of configurations appears to be converted from a "frozen" to a "melted" state by introduction of photoproducts.

Another remarkable observation: until now the positive dichroism has only been observed in the absence of bivalent ions; addition of $\mathrm{Mg}^{2+}$ is sufficient to convert the positive dichroism to the standard negative one. This result seems to be due to a reduction of the charge density of the DNA by $\mathrm{Mg}^{2+}$-binding and may also be partly due to an increase of the DNA flexibility in the presence of $\mathrm{Mg}^{2+}$. 


\section{Proteins: permanent anisotropy of the charge distribution}

\subsection{On the experimental assignment of permanent dipole moments}

The analysis of protein dipoles has a rather long tradition: Oncley and coworkers [32] studied protein dipoles by dielectric measurements already during the $30^{\prime}$ and $40^{\prime}$, long before any protein structure was known. Some of the dipole moments found by these measurements were unusually high and raised questions about their nature. It proved to be difficult to distinguish between different interpretations: do the electric moments reflect permanent anisotropies of the charge distribution or special forms of polarizabilities. A particular problem was raised by Kirkwood and Shumaker [33], who demonstrated by model calculations that fluctuations of the proton distributions in proteins may lead to considerable contributions to measured electric moments. A critical examination shows that an unequivocal assignment of electric parameters by dielectric measurements in their standard form is not possible. This is demonstrated, for example, by the fact that the electric parameters of DNA could not be distinguished from those of proteins. The experimental demonstration of the nature of a dipole moment clearly requires more than the measurement of dielectric increments at different frequencies. In this respect electrooptical measurements are clearly superior, provided that these measurements are performed at a sufficient time resolution: when the rise of the electric dichroism is analyzed with sufficient care, using well established criteria $[34,14]$, permanent dipoles may be distinguished from special forms of polarizabilities, including cases with a saturated polarizability like DNA double helices. Another advantage of the electrooptical approach is the fact that measurements can be extended up to high buffer concentrations, including physiological concentrations [35,36]. Most dielectric data were collected in aqueous solutions without buffer, which is more than dangerous for polyelectrolytes having structures that are strongly dependent on salt and proton concentrations.

\subsection{Examples of proteins with a permanent dipole moment}

Lac repressor The first case of a protein with a large permanent dipole moment in the authors laboratory [37] was found by accident during an analysis of the lac repressor. It has been known for a long time that lac repressor is a tetramer. For such a tetramer it was very suggestive to assume a symmetric tetrahedral structure, which should not have a permanent dipole moment. The experimental data, however, clearly demonstrated the existence of a huge permanent dipole moment of $1200 \mathrm{D}$ [37]. The evidence for the permanent moment did not only result from the dependence of the dichroism on the field strength, but more definitely from the time constants of the dichroism risecurves, which were in perfect agreement with the prediction of Benoit [34] in the limit of low field strengths. Thus, the conclusion could not be avoided that the structure of lac repressor is not symmetric, unless the data were perturbed by some special artifact. Almost ten years later the crystal structure of lac repressor has been solved and in fact the protein structure proved to be nonsymmetric [38-39]. A detailed comparison of experiments with calculations is not yet possible, because part of the lac repressor structure has not been resolved yet due to a high internal flexibility.

Lac repressor is an example of a DNA binding protein. Another example is the Tet-repressor, which also shows a large permanent dipole moment of $1050 \mathrm{D}$ [40]. The large dipole moment of the Tet-repressor is even more remarkable, because the molecular weight of the Tet-repressor is much lower than that of the lacrepressor. It is likely that the large dipole moments of these proteins are related to the DNA binding function: binding to the double helix with the large density of negatively charged phosphates is at least partly promoted by complementary positive charges at the protein binding site. Thus, the charge distribution of DNA binding proteins may be highly nonsymmetric in many cases.

$\alpha$-chymotrypsin proved to be an appropriate system for a detailed comparison of experiments and calculations. In this case the experimental data [41] showed a permanent dipole moment of $480 \mathrm{D}$ at $\mathrm{pH}$ 8.3; the dipole moment decreases with decreasing $\mathrm{pH}$ to $360 \mathrm{D}$ at $\mathrm{pH} 4.2$. Using the known crystal struc- 
ture it was possible to calculate a theoretical dipole moment from the coordinates of charged residues and from the partial charges associated with $\alpha$-helices. Because the protein bears a net charge at $\mathrm{pH}$-values other than the isoelectric one, the dipole moments had to be calculated with respect to the center of diffusion. The coordinates of the center of diffusion were determined by a bead model simulation based on the crystal structure. The dipole moments calculated according to this procedure at different $\mathrm{pH}$ values proved to be in agreement with the experimental one within the limits of accuracy of $\pm 10 \%$. Based on the crystal structure it was also possible to test the magnitude of contributions to the dipole moment resulting from fluctuations of the proton distribution. It could be shown that there is only a minor contribution resulting from these fluctuations in the case of chymotrypsin. For comparison it may be noted that dielectric measurements of $\alpha$-chymotrypsin provided a similar but not identical dipole moment [42].

Acetylcholinesterase (AChE) is a special case, which deserves a separate discussion. The crystal structure analysis of AChE from Torpedo californicus [43] demonstrated a distinct anisotropy of the charge distribution, which was used to calculate a dipole moment of $505 \mathrm{D}$ [44]. Because of a potential function of this dipole an experimental verification of this conclusion appeared to be desirable. This turned out to be more difficult than expected, because AChE from Torpedo californicus occurs as a dimer, where partial compensation of the moment must be expected. The problem has been solved by using a special AChE from the venom of a vietnamese snake, Bungarus fasciatus, which was demonstrated to occur as a monomer. Measurements demonstrated for this species a dipole moment of $1000 \mathrm{D}$ (in a $5 \mathrm{mM}$ MES buffer [45]). Because the amino acid sequence of the Bungarus AChE is similar but not identical with the Torpedo AChE, the Bungarus structure was modelled in analogy to the Torpedo structure. The dipole moment of $1830 \mathrm{D}$ calculated for this model, with respect to the center of diffusion, is higher than the experimental one. The difference between calculated and experimental values appears to be mainly due to the attachment of charged carbohydrate residues, which could not be included in the calculation.

The case of $\mathrm{AChE}$ is of interest for different reasons. First of all, it shows, how much calculated di- pole moments may deviate from the correct value, if not calculated with respect to the center of diffusion. AChE has an overall net charge of about 6 negative elementary charges. In such cases dipole moments must be calculated with respect to the center of diffusion, if the values should have any physical significance. The dipole moment of AChE is of special interest, because it may serve to direct the cationic substrate to the active site via a deep and narrow gorge. The dipole moment is parallel to the narrow gorge; model calculations suggest that the dipole moment contributes by guiding the substrate to the catalytic site [46] and, thus, increases the efficiency of this enzyme. AChE is characterized by an unusually high turnover rate.

Calculations of dipole moments are relatively simple, if the coordinates of crystal structures are available. Because large numbers of crystal structures may be easily obtained from e.g. the Brookhaven Protein Data Bank, there are now more results of calculations than reliable experimental data. The first compilation of calculated dipole moments has been presented by Barlow and Thornton [47] for 32 different proteins. Most of the dipole moments were in the range of several hundred Debye units, the largest value was $823 \mathrm{D}$. The most recent computation of 13 protein dipole moments includes a comparison of several procedures for the calculation [48]. It is remarkable that the results obtained by the most sophisticated procedure, the finite difference Poisson Boltzmann method, are not much different from those obtained by the most simple procedure, i.e. using equation (1) with standard pK-values and without consideration of interactions between ionizable sites. A similar conclusion was obtained previously in the case of $\alpha$ chymotrypsin [41]. During a screening of 500 protein crystal structures according to the simple procedure in the authors laboratory, most of the dipole moments were found in the range of several hundred Debye units; in 46 cases the dipole moment was more than $1000 \mathrm{D}$, in a few cases the dipole moment was even larger than $2000 \mathrm{D}$. These results illustrate the distribution of electric parameters in proteins; any potential functional implications have to be checked in each case separately. 


\subsection{Ionic strength dependence}

Because the structures of biological macromolecules are often strongly dependent on the ionic strength, the electric moments have been routinely measured at different salt concentrations. In most cases the resulting dipole moments showed very little dependence on the ionic strength. Recently measurements of the dipole moment of $\alpha$-chymotrypsin have even been extended up to ion concentrations of 0.1 M [36], using a special technique for electrooptical measurements at "physiological" salt concentration based upon cable discharge [35]. The permanent dipole moment remained even at this high salt concentration; its magnitude was reduced only by a relatively small amount (table 2 ). This raises the question, why the dipole moments of macromolecules are detectable in aqueous solutions, although the dipoles are expected to be shielded by the large number of counterions surrounding the macromolecules.

Table 2

Electrooptical data of $\alpha$-chymotrypsin obtained at $20^{\circ} \mathrm{C}$ at different salt concentrations. The data for the highest salt concentration are from [36], the other data are from [41]. Monovalent salt concentration $\mathrm{c}_{+}, \mathrm{Mg}^{2+}$-concentration $\mathrm{c}_{M g}$, limiting value of the electric dichroism $(\Delta \epsilon / \epsilon)_{\infty}$, dipole moment corrected for the internal field effect $\mu_{\text {curr. }}$ (cf. [41]).

\begin{tabular}{cccccc}
\hline$c_{+}$ & $\mathrm{c}_{M g}$ & $\mathrm{pH}$ & $(\Delta \epsilon / \epsilon)_{\infty}$ & $\mu_{\text {corr }}$ & $\mu_{\text {cor } r}$ \\
\hline$[\mathrm{mM}]$ & {$[\mathrm{mM}]$} & - & - & {$\left[10^{-27} \mathrm{Cm}\right]$} & {$[\mathrm{D}]$} \\
\hline 2.4 & - & 7.0 & 0.11 & 1.4 & 420 \\
7.4 & - & 7.0 & 0.13 & 1.4 & 420 \\
12.4 & - & 7.0 & 0.13 & 1.4 & 420 \\
9 & 0.2 & 8.3 & 0.10 & 1.5 & 450 \\
9 & 1 & 8.3 & 0.08 & 1.5 & 450 \\
19 & 1 & 8.3 & 0.05 & 1.7 & 510 \\
125 & 10 & 7.2 & 0.02 & 1.1 & 340 \\
\hline
\end{tabular}

The problem is analogous to that encountered with the electrophoretic motion of colloidal particles. Although the charge of the particles is shielded at some distance, nevertheless the particles move in electric fields. In these cases the electrophoretic motion is determined by the $\zeta$-potential at the plane of shear between the vesicle associated and the stationary part of the double layer [49-51]. The $\zeta$-potential has been defined for translational motion. The extension of this concept to rotational motion leads to the definition of a $\mu$-potential, which determines the torque at the plane of shear for rotational diffusion [52]. The thickness of the layer moving with the particles has been estimated to be in the order of $0.2 \mathrm{~nm}$ for the case of the $\zeta$-potential. It is very likely that the thickness of the corresponding layer in the case of the $\mu$-potential is of very similar magnitude. This interpretation provides a rationale for the relatively small dependence of experimental dipole moments on the salt concentration. A thin solvent layer associated with the protein molecules also explains the relatively close agreement of experimental dipole moments with those calculated from crystal structures.

\section{Membrane proteins}

\subsection{Electrostatics of bacteriorhodopsin}

Electrooptical measurements by various groups [53-56] provided evidence that bacteriorhodopsin disks are associated with permanent dipole moments in the range of 1 million of Debye. These unusually large dipole moments raised serious doubts about their nature. It has been suggested, for example, that at least part of the large dipole moment is of an "interfacial" character [57]. As discussed already above, it is not trivial at all to provide an unequivocal proof for the existence of a permanent anisotropy of the charge distribution in a biological macromolecule, because there are usually many alternative interpretations, which cannot be ruled out easily.

The electrooptical parameters of bacteriorhodopsin have been reinvestigated recently using stationary values of the dichroism measured over a broad range of electric field strengths [52]; these data (figure 5) have been analyzed in terms of the complete orientation function for disks having a permanent dipole moment in perpendicular direction to the plane and a polarizability parallel to the plane [58]. According to this analysis the permanent dipole moment is $4 \times 10^{6} \mathrm{D}$ for membrane fragments of $1 \mu$ diameter. Measurements on fragments of different size showed that the dipole increases with the area of the membrane disk. This result is consistent with a permanent anisotropy of the charge distribution and is hardly 


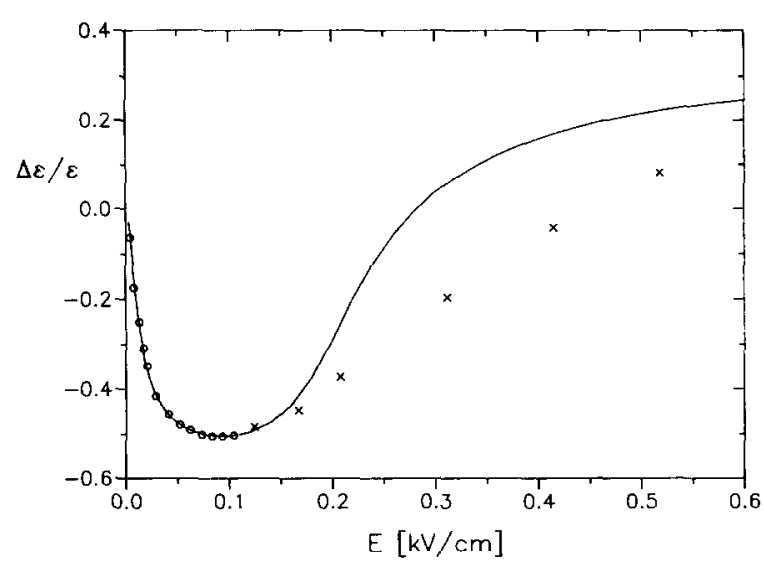

Fig. 5. Stationary reduced electric dichroism $(\Delta \epsilon / \epsilon)$ of bacteriorhodopsin as a function of the electric field strength $E$. The continuous line represents a least squares fit of the data points marked by (o) according to the orientation function of Shah [58] with a permanent dipole moment of $\mu=1.19 \times 10^{-23} \mathrm{Cm}$, a polarizability of $\alpha=6.6 \times 10^{-28} \mathrm{Cm}^{2} \mathrm{~V}^{-1}$ and a limiting value of the dichroism of $(\Delta \epsilon / \epsilon)_{\infty}=0.31$; the experimental data marked by $X$ were not included in the least squares fit; the deviations from the theoretical line at higher E-values seem to be due to the field induced changes discussed in section $6.2 .(1 \mathrm{mM} \mathrm{NaCl}$, $1 \mathrm{mM}$ Na-cacodylate $\mathrm{pH} 7.0,0.2 \mathrm{mM}$ EDTA; $20^{\circ} \mathrm{C}$, from [52]).

consistent with an "interfacial character". The polarizability in the plane of the disks, which is of an "interfacial character", increases with the 4th power of the disk radius [52].

The structure of bacteriorhodopsin has been determined by electron cryo-microscopy up to a resolution of $3.5 \AA$ in the direction of the membrane plane and of $4.3 \AA$ perpendicular to this plane [59]. The structure is not yet complete, because the ends of the peptide chains are "genuinely disordered"; nevertheless it is possible to calculate an approximate value of the dipole moment from this structure. The calculated value of $\sim 570 \mathrm{D}$ is a factor of 10 higher than the experimental value calculated per protein monomer ( $\sim 55 \mathrm{D})$. The large difference may be due to a nonsymmetric distribution of charged lipid residues.

According to the calculations the protein dipole is directed from the inside to the outside. There is also some experimental evidence for this direction [55]. It is well known that the function of bacteriorhodopsin is pumping of protons from the inside of cells to their outside [60-61]. This function requires supply of protons to the cytoplasmatic entrance of the pump and removal of protons from the exit. Obviously both supply and removal are based on diffusion. In both cases the efficiency may be increased by electrostatic gradients: at the entrance protons may be attracted by some negative potential, which may contribute to an increase of the target size by long range electrostatic interactions; at the exit the protons may be pushed away by some positive potential. The combined potentials are equivalent with a dipole moment directed from the inside to the outside and, thus, are consistent with the calculated direction of the dipole moment.

\subsection{Electrodynamics of bacteriorhodopsin}

A detailed comparison of the dichroism risecurves of bacteriorhodopsin with risecurves simulated for disks with equivalent hydrodynamic, electrical and optical parameters (figure 6) demonstrated that there is a relaxation process which cannot be attributed to the usual field induced rotation [52]. The additional process indicates a change of the bacteriorhodopsin structure, which is induced already at a relatively low electric field strength of $\sim 150 \mathrm{~V} / \mathrm{cm}$. The dependence of the time constant observed for the additional process indicates a very high change of the electric moment in the order of magnitude of $1 \times 10^{-24} \mathrm{Cm}$, corresponding to about $10 \%$ of the dipole moment for the whole disk; thus it is likely that the change involves the whole disk. The nature of the structure change is not clear yet. The field induced process is remarkable, because it represents an example for a structure change, which is induced already at a particularly low electric field strength.

\subsection{Electrostatics of $\mathrm{Na}^{+} / \mathrm{K}^{+}$-ATPase}

A recent investigation of $\mathrm{Na}^{+} / \mathrm{K}^{+}$-ATPase preparations by fluorescence detected measurements of its electric dichroism [62] demonstrated that its electric parameters are quite similar to those of bacteriorhodopsin (cf. [63]). The dependence of the dichroism on the electric field strength is again consistent with disks having a permanent dipole moment in the direction perpendicular to the plane and a high polarizability parallel to the plane. The permanent dipole moment for the disks is in the range of $10^{6} \mathrm{D}$; the dipole moment per ATPase monomer is estimated to be $430 \pm 50 \mathrm{D}$. Thus, $\mathrm{Na}^{+} / \mathrm{K}^{+}$-ATPase represents another case of a membrane protein, where the organi- 


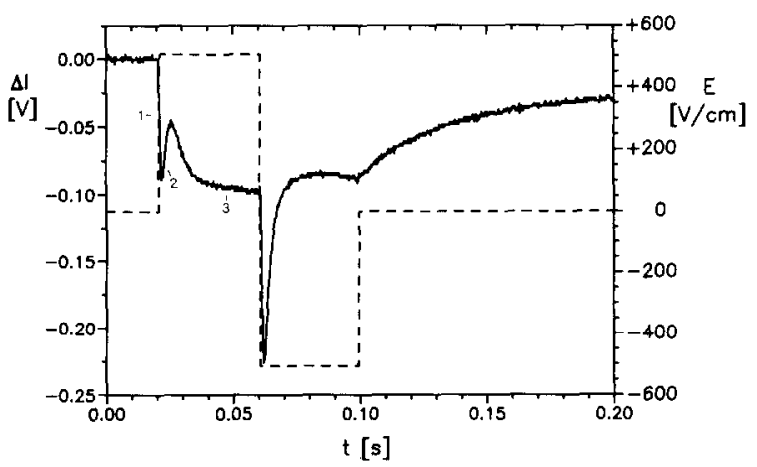

(a)

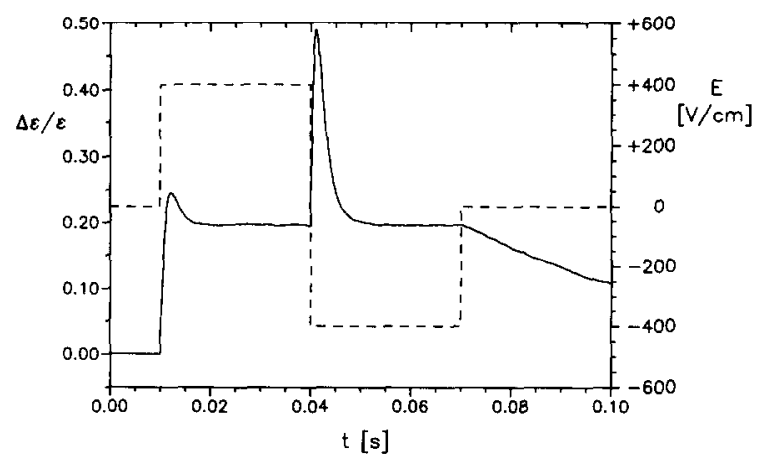

(b)

Fig. 6. Comparison of experimental (a) and computed (b) dichroism rise curve of bacteriorhodopsin at an intermediate electric field strength $\mathrm{E}$ (from [52]). (a) Change of the light intensity $\Delta I$ transmitted by a bacteriorhodopsin solution induced by an electric field pulse $\mathrm{E}$ (dashed line); the light $(\lambda=546 \mathrm{~nm}$ ) was polarized parallel to the field vector; $1 \mathrm{mM} \mathrm{NaCl}, 1 \mathrm{mM} \mathrm{Na}$-cacodylate $\mathrm{pH}$ $7.00 .2 \mathrm{mM}$ EDTA; $20^{\circ} \mathrm{C}$; the exponential processes in the rise curve are indicated by 1,2 and 3: (b) Brownian dynamics simulation of the electrooptical transient of a disk with an effective diameter of $980 \mathrm{~nm}$ (diffusion tensor obtained by bead model simulation) using the following electric parameters: permanent dipole perpendicular to the plane $8.7 \times 10^{-24}$; polarizability parallel to the plane $4.9 \times 10^{-28} \mathrm{Cm}^{2} \mathrm{~V}^{-1}$; extinction tensor: in plane components 76000 , component perpendicular to the plane 19000: Note that the transmitted light intensity is shown in (a), whereas $(\Delta \epsilon / \epsilon)$ is shown in (b); this is the reason for the amplitude inversion; the relaxation process 3 is clearly visible in (a) but not in (b). zation of a large number of monomers in a membrane patch leads to an extremely high dipole moment. It is very likely that this property is related to the vectorial function of the protein (cf. 6.1).

\section{Symmetry breaking in mixed lipid vesicles}

Usually the distribution of charged molecules is regulated by electrostatic interactions and thus accumulation of charged residues of a given type, associated with a large anisotropy of the charge distribution, is avoided. However, under special conditions this rule may be violated to some degree due to the existence of special molecular interactions. A case of this type has been observed for mixed lipid vesicles.

Lipid bilayers may be composed of many different lipid components. For simplicity, it may be assumed that a given bilayer is composed of a 1/1 mixture of one lipid component bearing a net charge and another one without a net charge. When the mixture is at a sufficiently high temperature, the two components will be mixed homogeneously within the bilayer. If the bilayer is organized in the form of a spherical vesicle, the vesicle is expected to be without an electric dipole moment, as long as the lipids in the sphere are distributed homogeneously.

If the temperature of the solution containing the vesicles is decreased, separation of the lipid components into separate phases must be expected. The type of separation is dependent on the magnitude of the interactions between the components. For simplicity it may be assumed that there is a high cooperativity of the phase separation leading to a complete separation of the components: in this case one of the components is driven into one half of the sphere and the other one into the other half. It should be emphasized that this model represents an extreme case; separation cannot be expected to be complete in practice. However, the extreme case may be used for a simple model calculation: after phase separation there is a high anisotropy of the charge distribution. Based on integration using polar coordinates ([64], cf. figure 7) the dipole moment is expected to be

$$
d_{z}=q_{d} 2 \pi r^{3} \int_{0}^{\pi / 2} \cos (\varphi) \sin (\varphi) d \varphi=q_{d} \pi r^{3}
$$



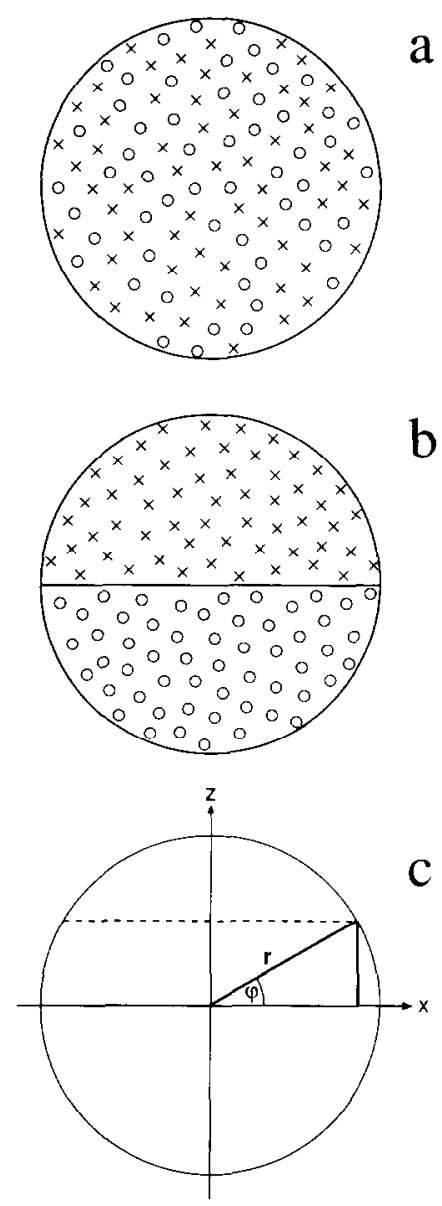

Fig. 7. Scheme of the phase separation in a spherical vesicle with two different lipid components: a) symmetric distribution before phase separation; b) limit case of complete phase separation; c) calculation of the dipole moment under the assumption that the charged lipids are segregated into the upper half of the sphere (from [64]).

where $q_{d}$ is the effective charge density in the half of the sphere containing the charged residues and $r$ is the radius of the lipid vesicle.

For a double layer with a lipid packing density of $\sim 2$ molecules $/ \mathrm{nm}^{2}$ in each layer [65-66] and using the assumption of a reduced charge density corresponding to $\sim 10 \%$ of the phosphate density, the charge density is approximately $q_{d} \approx 6 \times 10^{-20} \mathrm{C} / \mathrm{nm}^{2}$; in this case the dipole moment for a vesicle with a radius $r=$ $100 \mathrm{~nm}$ is $\sim 6 \times 10^{7} \mathrm{D}$. As mentioned above, this estimated value represents an upper limit.

For an experimental investigation lipid vesicles have been prepared from 50\% dimyristoyl-1- $\alpha$-phosphatidylcholin (DMPC) and 50\% dimyristoylphophatidic acid (DMPA). The experimental data, including transients measured with reversal of electric field pulses, indicate the existence of a permanent dipole moment at temperatures below $30^{\circ} \mathrm{C}$. These indications have not been observed for vesicles prepared from DMPC or DMPA alone and also have not been observed at temperatures above $30^{\circ} \mathrm{C}$. According to a simple interpretation of light scattering amplitudes measured as a function of the electric field strength by the orientation function for permanent dipoles, the permanent dipole moment for vesicles with an average diameter of $\sim 200 \mathrm{~nm}$ is of the order of magnitude of $\sim 2.4 \times 10^{6} \mathrm{D}$ [64].

\section{Conclusions}

The electric parameters and the field induced processes of biological macromolecules are of unusually high complexity because there is a wide range of different structures and most of these structures bear large numbers of charged residues. In addition, most of the structures are subject to changes between different states. Under these conditions the correct assignment of experimental data may be rather difficult. However, the problems may be solved by careful experiments combined with calculations on appropriate models.

In the case of proteins, for example, it has been rather difficult in the past to rule out that the major part of dipole moments results from proton fluctuations. A detailed interpretation of experimental data may be supported by model calculations based on crystal structures. Using the coordinates of charged residues, it is relatively simple to calculate dipole moments, including e.g. contributions resulting from proton fluctuations. Moreover, information about the direction of dipole moments may be obtained from values of the electric dichroism and may also be used for a comparison with corresponding information derived from crystal structures; this may be essential for a decision between different interpretations. Meanwhile a close correspondence between calculated and measured dipole moments has been demonstrated for a rather large number of cases; usually the dipole moment is mainly determined by the anisotropy of residues bearing full charges, i.e. lysine, arginine, 
aspartate and glutamate residues. It should be noted that the calculated result for molecules with a net charge, may be rather far away from the correct one, if the reference is not the center of diffusion (cf. 5.2). Dipole moments of several hundred Debye appear to be standard among proteins, whereas dipole moments in the range above 1000 Debye are less common, but have been observed already in several cases.

For membrane proteins organized in large patches, the relatively small dipole moments associated with individual molecules add up to huge moments of several $10^{6}$ Debye for the whole patch. It is likely that these dipole moments support vectorial transport across the membrane by attracting ions on one side and repulsion of these ions on the other side.

The problems associated with the assignment of electric moments have been quite obvious for the case of nucleic acids, where unusual electrooptical effects have been reported by several authors. The interpretation of rather puzzling effects has been possible by detailed model calculations; all the parameters introduced into these calculations are on the basis of the essential features of nucleic acid structure; there is no conflict with any established parameter. Now, the special effects can be used to learn about the physical properties of nucleic acids, e.g. the dynamics of double helices.

Electric parameters of unusual magnitude have been reported in the literature in a rather large number of cases. Usually these observations remained unexplained. The main challenge is the appropriate assignment in terms of reasonable models. In recent years the assignment has been advanced for an increasing number of cases. The acquired knowledge should be useful, for example, for a judgement on bioelectric phenomena. Questions on the physical basis of electric field sensors with a particularly high sensitivity are directly related to the electric parameters of biological macromolecules. Some of the systems discussed in the present review demonstrate the possibility of field induced reactions at electric field strengths, which are lower than anticipated previously. Thus, it may be expected that further extensions in this direction are possible.

\section{Acknowledgements}

The generous support by Manfred Eigen during many years in his department "Biochemische Kinetik" is gratefully acknowledged.

\section{References}

[1] W. Heiligenberg, in Neurowissenschaft, J. Dudel, R. Menzel and R.F. Schmidt, Editors, Springer; Berlin (1996) 427-435

[2] J.C. Weaver and R.D. Astumian, Science 247 (1990) 459 462.

[3] M. Eigen and L. DeMayer, in Techniques of Organic Chemistry, S.L. Friess, E.S. Lewis and A. Weissberger, Editors, Vol. 8, Part II, Interscience New York (1963) 895-1045.

[4] C. Bernasconi, Relaxation Kinetics, Academic Press, New York (1976)

[5] D. Porschke, Ber. Bunsenges. Phys. Chem. 100 (1996) 715 720.

[6] J. Garcia de la Torre and V.A. Bloomfield, Q. Rev. Biophys. 14 (1981) $81-139$.

[7] J. Antosiewicz and D. Porschke, J. Phys. Chem. 93 (1989) 5301-5305.

[8] K.J. Mysels, J. Chem. Phys. 21 (1953) 201-205.

[9] J. Antosiewicz and D. Porschke, Biophys. Chem. 33 (1989) 19-30.

[10] W.A. Wegener, R.M. Dowben and V.J. Koester, J. Chem. Phys. 70 (1979) 622-623.

[11] W.A. Wegener, J. Chem. Phys. 84 (1986) 5989-6004.

[12] J. Antosiewicz and D. Porschke, J. Phys. Chem. 97 (1993) 2767-2773.

[13] U. Mohanty and Y. Zhao, Biopolymers 38 (1995) 377-388.

[14] E. Fredericq and C. Houssier, Electric Dichroism and Electric Birefringence, Clarendon Press, Oxford (1973).

[15] M. Hogan, N. Dattagupta and D.M. Crothers, Proc. Natl. Acad. Sci. USA 75 (1978) 195-199.

[16] N.C. Stellwagen, Biopolymers 20 (1981) 399-434.

[17] S. Diekmann, W. Hillen, M. Jung, R.D. Wells and D. Porschke, Biophys. Chem. 15 (1982) 157-167.

[18] K. Yoshioka, J. Chem. Phys. 79 (1983) 3482-3486.

[19] S. Diekmann, M. Jung and M. Teubner, J. Chem. Phys. 80 (1984) 1259-1262.

[20] J.G. Elias and D. Eden, Macromolecules 14 (1981) 410-419.

[21] D.C. Rau and E. Charney, Biophys. Chem. 14 (1981) 1-9.

[22] M. Eigen and G. Schwarz, in "Electrolytes", Pergamon Press, New York (1962) 309-335.

[23] N. Ise, M. Eigen and G. Schwarz, Biopolymers 1 (1963) 343-352.

[24] D. Porschke and G. Nolte, Macromolecules 27 (1994) 590595.

[25] D. Porschke, Biophys. Chem. 51 (1994) 37-43.

[26] D. Porschke, Biophys. Chem. 22 (1985) 237-247.

[27] M. Yoshioda, K. Kikuchi, T. Maekawa and H. Watanabe, J. Phys. Chem. 96 (1992) 2365-2371. 
[28] T. Grycuk, J. Antosiewicz and D. Porschke, J. Phys. Chem. 98 (1994) 10881-10887.

[29] M. Fixman and S. Jagannathan, J. Phys. Chem. 75 (1981) 4048-4059.

[30] J. Antosiewicz and D. Porschke, J. Phys. Chem., in press

[31] D. Porschke, Biophys. Chem. 49 (1994) 127-139.

[32] J. J. Oncley, in J.J. Cohn and J.T. Edsall (Editors), Proteins, Amino Acids and Peptides, Am. Chem. Soc. Monograph Series (1943)

[33] J.G. Kirkwood and J.B. Shumaker, Proc. Natl. Acad. Sci. USA, 38 (1952) 855-862.

[34] H. Benoit, Ann. Phys. 6 (1951) 561-609.

[35] D. Porschke and A. Obst, Rev. Sci. Instr. 62 (1991) 818-820.

[36] T. Schönknecht and D. Porschke, Biophys. Chem. 58 (1996) 21-28.

[37] D. Porschke, Biophys. Chem. 28 (1987) 137-147.

[38] A.M. Friedman, T.O. Fischmann and T.A. Steitz, Science 268 (1995) 1721-1727.

[39] M. Lewis, G. Chang, N.C. Horton, M.A. Kercher, H.C. Pace, M.A. Schumacher, R.G. Brennan and P. Lu, Science 271 (1996) 1247-1254.

[40] D. Porschke, K. Tovar and J. Antosiewicz, Biochemistry 27 (1988) 4674-4679.

[41] J. Antosiewicz and D. Porschke, Biochemistry 28 (1989) $10072-10078$.

[42] S. Takashima, J. Phys. Chem. 100 (1996) 3855-3860.

[43] J.L. Sussman, M. Harel, F. Frolow, C. Oefner, A. Goldman, L. Toker and I. Silman, Science 253 (1991) 872-879.

[44] D.R. Ripoll, C.H. Faerman, P.H. Axelsesen, I. Silman and J.L. Sussman, Proc. Natl. Acad. Sci. USA 90 (1993) 51285132.

[45] D. Porschke, C. Creminon, X. Cousin, C. Bon, J. Sussman and I. Silman, Biophys. J. 70 (1603-1608.

[46] J. Antosiewicz, M.K. Gilson, I.H. Lee and J.A. McCammon, Biophys. J. 68 (1995) 62-68.

[47] D.J. Barlow and J.M. Thornton, Biopolymers 25 (1986) $1717-1733$

[48] J. Antosiewicz, Biophys. J. 69 (1995) 1344-1354.

[49] H. Helmholtz, Ann. Physik 7 (1879) 337-382.

[50] J.T. Overbeek and P.H. Wiersena, in "Electrophoresis - Theory, methods and applications", M. Bier, Editor, Academic Press, New York (1967) 1-52.

[51] S. McLaughlin, in "Current Topics in Membranes and Transport" Vol. 7, F. Bronner and A. Kleinzeller, Editors, Academic Press, New York (1977) 71-144.

[52] D. Porschke, Biophys. J. 71 (1996) 3381-3391.

[53] L. Keszthelyi, Biochim. Biophys. Acta 598 (1980) 429-436.

[54] S. Druckmann and M. Ottolenghi, Biophys. J. 33 (1981) 263-268.

[55] Y. Kimura, M. Fujiwara and A. Ikegami, Biophys. J. 45 (1984) 615-625.

[56] K. Barabás, A. Dér, Z. Dancsházy, P. Ormos, L. Keszthelyi and M. Marden, Biophys. J. 43 (1983) 5-11.

[57] S.G. Taneva, N. Jordanova and I.B. Petkanchin, Biophys. Chem. 44 (1992) 91-97.

[58] M.J. Shah, J. Phys. Chem. 67 (1963) 2215-2219.
[59] N. Grigorieff, T.A. Ceska, K.H. Downing, J.M. Baldwin and R. Henderson, J. Mol. Biol. 259 (1996) 393-421.

[60] W. Stoeckenius, R.H. Lozier and R.A. Bogomolni, Biochim. Biophys. Acta 505 (1979) 215-278.

[61] J.K. Lanyi, Biochim. Biophys. Acta 1183 (1993) 241-261.

[62] D. Porschke and E. Grell, Biochim. Biophys. Acta 1231 (1995) 181-188.

[63] C. Gergely, A. Dér, S. Száraz and L. Keszthelyi, Bioelectrochem. Bioenerg. 28 (1992) 149-157.

[64] D. Porschke, J. Phys. Chem. 100 (1996) 472-473.

[65] G. Cevc and D. Marsh, Phospholipid bilayers - Physical Principles and Models, Wiley Interscience, New York (1987).

[66] R.B. Gennis, Biomembrancs - Molecular Structure and Function, Springer, Berlin (1989). 